\title{
End-to-end simulations of different coronagraphic techniques
}

\author{
John E. Krist ${ }^{\mathrm{a}}$, Dwight C. Moody ${ }^{\mathrm{a}}$, Dimitri Mawet ${ }^{\mathrm{a}}$, John T. Trauger ${ }^{\mathrm{a}}$, Ruslan Belikov ${ }^{\mathrm{b}}$, Stuart K. \\ Shaklan ${ }^{\mathrm{a}}$, Olivier Guyon ${ }^{\mathrm{c}}$, Robert J. Vanderbei ${ }^{\mathrm{d}}$ \\ ${ }^{a}$ Jet Propulsion Laboratory, California Inst. of Technology, 4800 Oak Grove Dr., Pasadena, CA \\ 91109; ${ }^{b}$ NASA Ames Research Center, Moffet Field, CA; ${ }^{\mathrm{C}}$ National Optical Observatory of

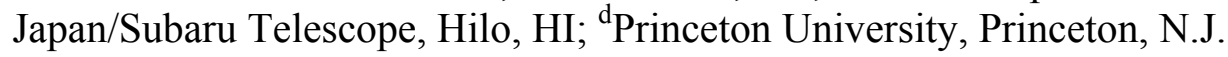

\begin{abstract}
The NASA exoplanet exploration program is dedicated to developing technologies for detecting and characterizing extrasolar planets. In support of that program we have evaluated three different coronagraphic techniques (bandlimited Lyot, optical vortex, and phase-induced pupil apodization) using optical propagation simulations. These utilized a complete hypothetical telescope+coronagraph system with phase and amplitude aberrations. Wavefront control using dual sequential deformable mirrors was performed. We discuss the different computational techniques necessary to accurately simulate each coronagraph.
\end{abstract}

Keywords: coronagraph, optical modeling, bandlimited coronagraph, optical vortex coronagraph, PIAA

\section{INTRODUCTION}

\subsection{Coronagraphs}

The direct imaging of extrasolar planets requires a high contrast imaging system comprising a telescope, a wavefront control system with at least one deformable mirror (DM) to reduce scatter generated by optical errors, and a coronagraph to suppress the diffraction pattern of the star. Such a system must achieve planet/star brightness contrast ratios of $10^{-9}$ for Jupiter-like planets and $10^{-10}$ for Earths in visible light. It must also have a small Inner Working Angle (IWA), which is the smallest angle at which the planet can be seen distinct from the star. Most planet imaging systems are being optimized for IWAs of 2-3 $\lambda / D$ radians ( $D=$ telescope diameter). The telescope, DMs, and coronagraph, along with the wavefront sensing system that is used to modify the DMs, must be considered a single system. Each coronagraph responds differently to wavefront errors, which can affect the level of wavefront correction possible. Thus, their realworld performance limits cannot be assessed without including the entire optical system. Analytical computations that consider only their diffraction suppression performance in an aberration-free system may therefore be misleading.

We have selected three coronagraph types for this study: the hybrid bandlimited Lyot, optical vector vortex, and phase induced amplitude apodization. These all have fourth-order aberration tolerances, have throughputs greater than $30 \%$, and allow imaging to within $2-3 \lambda / D$ of the star.

\subsection{Hybrid bandlimited Lyot coronagraph}

The Bandlimited Lyot Coronagraph ${ }^{1}$ (BLC) is a version of the classical Lyot coronagraph, which has a mask (occulter) at an intermediate focus that blocks light from the star and an aperture stop (Lyot stop) at a subsequent image of the pupil. A BLC uses an occulter that has a grayscale transmission pattern that filters the unblocked starlight so that it is fully concentrated around the edge of the pupil in the reimaged pupil plane. This allows a simple aperture stop to mask the outer pupil, completely suppressing the diffracted light from the occulted point source (in an aberration-free system). Light from unocculted sources, such as planets or circumstellar disks, can pass through with small attenuation.

A problem with the amplitude-only occulter in a classical BLC is that is has low throughput when designed to allow imaging close to the star because the Lyot stop must be small. A new variety, the hybrid BLC ${ }^{2}$ (HBLC), uses an occulter combining a grayscale amplitude transmission occulter with a phase-modulating pattern consisting of dielectric coatings. Together these allow for higher throughput by allowing the use of a more open Lyot stop. The dielectric coatings can also be optimized to reduce the wavelength-dependent phase dispersion introduced by amplitude-modulating pattern.

Techniques and Instrumentation for Detection of Exoplanets IV, edited by Stuart B. Shaklan, Proc. of SPIE Vol. 7440, $744016 \cdot$ ? 2009 SPIE · CCC code: 0277-786X/09/\$18 · doi: 10.1117/12.826487 


\subsection{Optical Vector Vortex coronagraph}

An optical vortex ${ }^{3}(\mathrm{OV})$ is formed when a plane wave encounters a surface that introduces an azimuthally-varying phase retardance, causing one side of the wavefront to lag the opposite side (a "phase screw"). OVs are useful in extrasolar planet imaging because they can be used in place of amplitude occulting masks in a Lyot coronagraph while providing a similar effect. In theory they allow imaging closer to the star and with a low reduction in throughput due to a more open Lyot stop when compared to most other coronagraphic techniques.

The problem with OVs is that they are difficult to make. Early methods created etched screw-shaped surface profiles in an optical substrate ${ }^{4}$. However, inevitable fabrication errors, especially at the singularity at the center of the screw, make these untenable for very high contrast applications (they also have poor broadband performance). A recent alternative method for producing a vortex utilizes precisely applied coatings that azimuthally modify the polarization of light in the wavefront ${ }^{5}$. The phase ramps can be more accurately created in this manner. There are still some errors introduced at the center of the phase screw, which are blocked by applying a small $(<1 \lambda / \mathrm{D})$ disk on top of the coatings. This is called an Optical Vector Vortex Coronagraph (OVVC).

\subsection{Phase induced amplitude apodization}

It is well know that apodizing the pupil of a telescope can suppress the wings of the diffraction pattern. This can be done by inserting a pattern at an intermediate pupil that tapers the transmission towards the edge of the beam. However, doing this comes at the expense of a larger PSF core (and more blurred planets) and a great reduction in throughput. It is also difficult to make an apodizer that is sufficiently accurate and operates over a broad wavelength range for extrasolar planet imaging. The Phase Induced Amplitude Apodization ${ }^{6}$ (PIAA) method, in theory, produces equivalent apodizations without a large loss in throughput or resolution.

PIAA utilizes two optics with unconventional surface profiles. The first optic is located at a pupil plane and performs the apodization by remapping the beam to create the desired concentration of light on the second optic. The second compensates for the large phase errors this introduces, creating a flat wavefront. The shapes of the optics, especially the first, are unconventional. On the first optic the curvature varies rapidly toward the edge in order to produce the apodization. In a two-optic PIAA system, this can introduce diffraction effects that reduce contrast and broadband performance as well as require unachievable tolerances on the fabrication. To reduce these effects, a mild apodizer is placed after the second optic, making the curvature on the first more practical. This decreases throughput by $10 \%-20 \%$, but this is much less than would occur by using only an amplitude apodizer.

The apodized beam is brought to focus, creating an image of the star with a broad core but dramatically reduced wings, so the star's image is blocked by a simple hard-edge occulter. The remapped wavefront causes a distortion of the spatial coordinates, and an off-axis source appears highly comatic. After the occulter the beam is sent through another set of PIAA optics in reverse order, correcting for both effects.

\section{MODELING}

End-to-end modeling of a coronagraph involves computing the propagation of a wavefront through a realistic optical system, sensing the wavefront at the image plane, deriving settings for the deformable mirrors to create a dark field around the star in the image, and then computing the final image using those settings. During this process, the performance of a given optical configuration can be evaluated in terms of sensitivity to and ability to control wavefront errors over a given bandpass. From this, requirements can be set on critical optical surfaces and telescope pointing.

\subsection{Optical system design}

We have simulated a complete coronagraphic optical system comprising a telescope, dual deformable mirrors for wavefront control, and a selection of coronagraphs. We have assumed a Gregorian off-axis, unobscured system with a circular primary mirror. There are two separate configurations: the hybrid Lyot and optical vortex coronagraphs share the same system as they both use image and pupil plane masks that can be made selectable, while PIAA requires a number of additional optics to accommodate the forward and reverse PIAA systems. The first few optics in each configuration are the same and it would be possible to make the coronagraphs selectable modes via flip mirrors. The layouts are shown in Figures 1 and 2.

Note that the physical diameter of the optics is not relevant to the results presented in this study as long as the spatial resolution on the sky is described in parameters that scale with the telescope (e.g. $\lambda / D$ radians) and the distances between 
optics scale with diameter. For reference, at $\lambda=550 \mathrm{~nm}, 1.0 \lambda / D$ radians is equivalent to 75 milliarcseconds $(D=1.5 \mathrm{~m})$, 28 mas $(D=4 \mathrm{~m})$, or 14 mas $(D=8 \mathrm{~m})$.

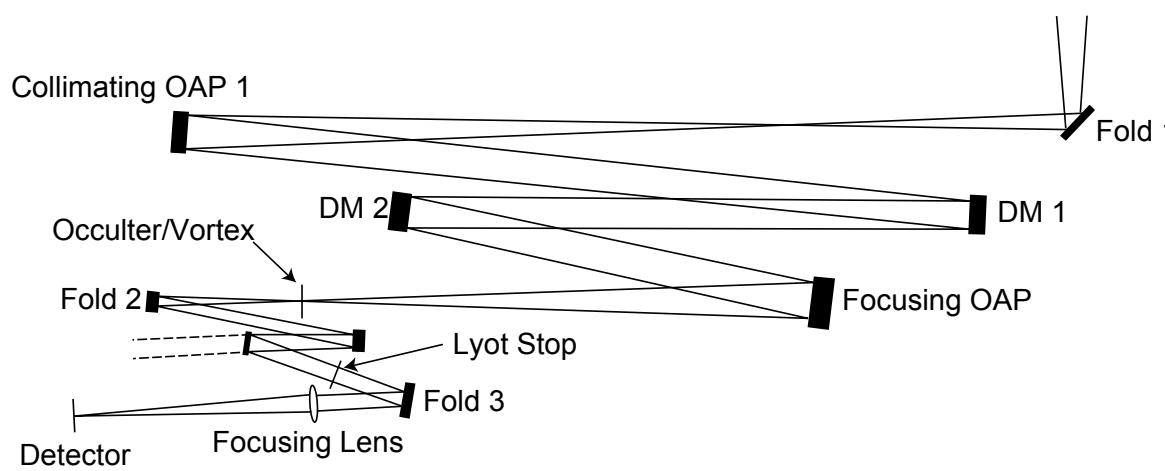

Fig. 1. Schematic layout of the optical system for the bandlimited Lyot and optical vortex coronagraphs. Not shown are the primary and secondary mirrors. Light from the telescope enters from the top right corner. A dichroic beam splitter before the Lyot stop allows the beam to be split into separate wavelength channels, each with its own Lyot stop.
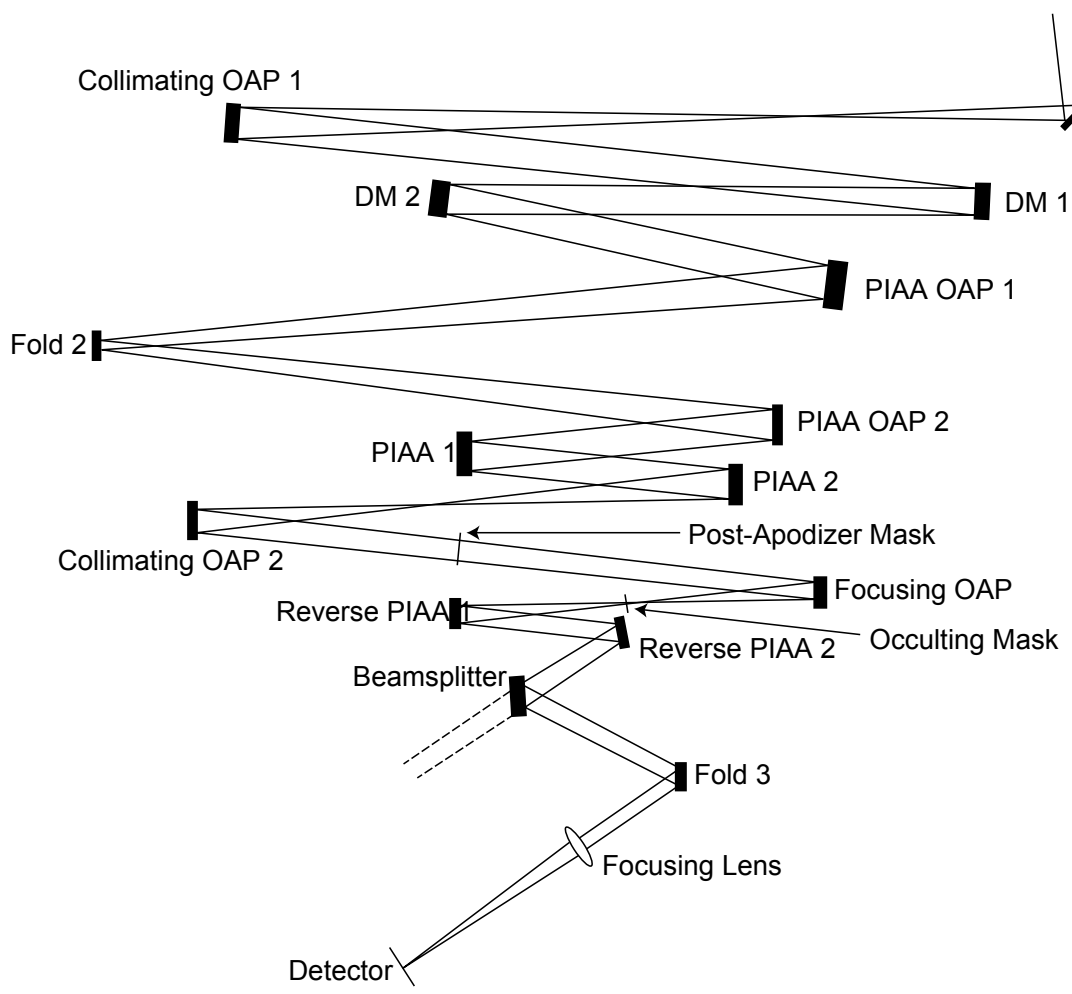

Fig. 2. Schematic layout of the optical system for the PIAA coronagraph. Not shown are the primary and secondary mirrors. Light from the telescope enters from the top right corner. A dichroic beam splitter before the Lyot stop allows the beam to be split into separate wavelength channels, each with its own Lyot stop.

\subsection{Wavefront propagation software}

The accurate modeling of how the wavefront changes as it passes through the optical system requires algorithms that compute the effects of diffraction and scattering at each surface. Fraunhofer (far-field) diffraction, which can simply be computed by the Fourier transform of the wavefront, may be used when propagating directly from a pupil plane (e.g., the primary mirror) to an image plane and vice-versa. However, it does not account for the effects of intermediate optics that are important to high contrast coronagraphic imaging.

As the wavefront propagates, phase errors gradually transform into amplitude errors and vice-versa (the Talbot effect ${ }^{7}$ ). A surface height deviation in an optic, perhaps caused by imperfect polishing, will alter the wavefront's phase. If that 
optic is located somewhere other than a pupil or image plane, the phase error will turn into a combination of phase and amplitude errors by the time the wavefront reaches the detector. Scattered light in the image plane behaves differently with wavelength depending on whether it was created by a phase or amplitude error, and the two types of errors are corrected differently. The correction of amplitude errors in the image plane actually makes use of the Talbot effect by altering the wavefront's phase with a deformable mirror located at an intermediate plane ${ }^{8}$. Thus, it is critical to use more complex algorithms that can handle optics at intermediate (near-field) planes when modeling high contrast systems. For most systems, Angular Spectrum and Fresnel propagation algorithms suffice ${ }^{7}$.

For this study we have applied two different optical modeling packages that utilize the same near-field and far-field propagation algorithms and produce similar results. The first is proprietary code that has been used extensively for simulating coronagraphs in the High Contrast Imaging Testbed (HCIT) at the Jet Propulsion Laboratory (JPL) and has been verified against actual HCIT results. It was used for simulating the bandlimited occulter Lyot coronagraphs in this study. The other package is the PROPER ${ }^{9}$ optical propagation library for IDL. PROPER is free and publicly available from www.openchannelsoftware.com. PROPER has routines that compute both near and far-field diffraction using automatically-chosen algorithms. It can create amplitude and phase errors on each optic specified by Zernike polynomials, power spectra, or user-provided maps. It also includes a model of a Xinetics deformable mirror with measured actuator influence functions. PROPER was used to simulate the optical vortex and PIAA coronagraphs in this study. The proprietary JPL software has similar routines.

Both packages compute the wavefront through an unfolded system (i.e., no tilted optics), so for these simulations the components were extracted from the Zemax optical prescription and placed along a single axis. Mirrors were converted to equivalent thin lenses. The same unfolded layout was used for the bandlimited and optical vortex coronagraphs. The PIAA system, which has a number of additional components, shared only the first few optics and required a largely different layout. Only the V-band channel was modeled.

Propagation within the bandlimited Lyot, optical vortex, and shaped pupil coronagraphs can be modeled using standard algorithms, though the representation of the vortex mask is complicated. PIAA, however, requires custom algorithms to propagate between the remapping optics, as detailed later.

\subsection{Wavefront representation}

The wavefront in the simulations is represented by a $2048 \times 2048$ double-precision complex-valued array. This size is sufficient to accommodate the highest spatial frequency surface errors of concern and can be efficiently processed on the available computers. All of the simulations produce sampling in the image plane that is better than the Nyquist criterion $(0.5 \lambda / D$ radians per pixel). This is accomplished by defining the entrance pupil diameter to be less than half the wavefront array diameter. In the case of the optical vortex, the image sampling is even finer $(0.1 \lambda / D$ per pixel).

\subsection{Occulter definition}

Each coronagraphic system has an image plane occulter. The hybrid bandlimited Lyot coronagraph occulter has a greyscale amplitude transmission profile as well as a phase-modulating coating that provides a $4^{\text {th }}$ order aberration tolerance. The occulter profile is defined to have $50 \%$ intensity transmission at a radius of 2 or $3 \lambda / D$ radians at the central wavelength $(550 \mathrm{~nm})$. The PIAA occulter is a hard-edge spot located at the focus of the PIAA mirrors. It produces a $50 \%$ transmission at a radius of $2.5 \lambda / D$. The optical vortex has a phase mask of charge $=4\left(4^{\text {th }}\right.$ order aberration tolerance) with a small hard-edge spot at the center with a radius of $0.25 \lambda / D$.

\subsection{Dark hole size}

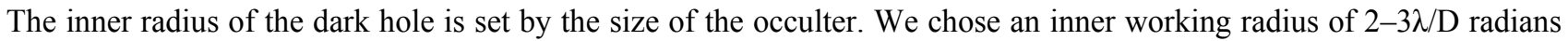
at the central wavelength $(550 \mathrm{~nm})$, depending on the coronagraph. However, the effective IWA is not fully a function of the coronagraph, as wavefront control at the lower spatial frequencies corresponding to these radii is critical to achieving the required contrast. The outer radius is limited by the number of DM actuators that span the pupil diameter. If a DM has $\mathrm{N}$ actuators across the pupil, the maximum region that it can correct is $\mathrm{N} \lambda / \mathrm{D}$ radians in diameter. In practice, deep contrast fields can only be achieved using even smaller outer limits. In this study we use DMs having 48 actuators across the pupil diameter and limit the outer radius of the dark hole to be equal to $20 \lambda / \mathrm{D}$ at the shortest wavelength.

\subsection{Optical fabrication errors}

A two-dimensional map of surface errors was generated for each optic in the system (Figure 3). The spatial frequency distribution of the errors was defined by power spectral density (PSD) curves derived from actual high-quality optics. 
The primary mirror was set to have an RMS surface error of $7.5 \mathrm{~nm}$. The other optics were set to have $1.25 \mathrm{~nm}$ RMS surface errors. Such high-quality surfaces, even on off-axis aspherical mirrors, are well within the capability of commercial fabricators (e.g., Tinsley, ASML, etc.). During our modeling it became apparent that certain components (flat mirrors near focus and the mirrors between the PIAA optics and occulter) required even lower errors in order to achieve the necessary $10^{-9}$ contrast performance. At these critical locations phase errors can create large amplitude errors that are impossible to fully correct in broadband light. Measured surface error maps of PIAA optics created by Tinsley were used for the two forward PIAA mirrors.

Coating errors that cause non-uniform reflection can also reduce the contrast performance. Because of its size, the primary mirror represents the most difficult optic to coat uniformly. We generated a synthetic reflectivity error map for the primary based on a reflectance PSD derived from test samples that were distributed within a large-optic coating chamber at ITT. We assumed that the uniformity on the smaller optics was not important and thus did not include reflectivity maps for them.

Simulation of the PIAA system requires that the wavefront be distorted by the forward PIAA and undistorted by the reverse PIAA. This is implemented via interpolation. During PIAA remapping, the wavefront errors are spatially compressed near the beam axis. In order to prevent aliasing from high spatial frequency aberrations during the forward and inverse remapping steps, the surface and primary reflectivity maps were filtered to a maximum spatial frequency of 45 cycles across the beam diameter ( 85 cycles for the optics between the forward PIAA and occulter). Thus, at the final focus the uncorrected scattered light caused by these errors can be seen extending out to $45 \lambda / D$ radians. This is sufficient to encompass high spatial frequency errors that through folding and other effects scatter light into the $20 \lambda / D$ radius dark hole region.

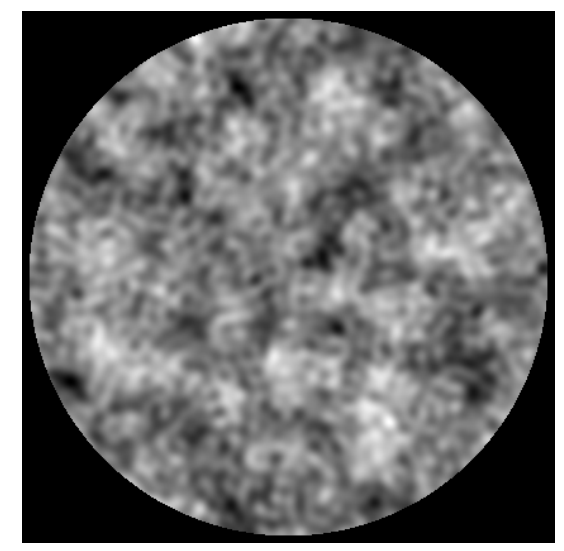

Fig. 3. Synthetic phase error map of the primary mirror surface used in this study.

\subsection{Wavefront control}

The simulation software is used to generate images at the final focus of the system. The wavefront can be derived from such images either by: (1) introducing a variety of known wavefront disturbances (e.g., altering the DM surface with specific patterns), measuring the image plane intensities, and solving for the common wavefront; or (2) directly using the complex electric field computed by the propagation software. Option (1) represents what would be used for a real system in which only wavefront intensity can be measured by the detector. Our experience shows that both methods produce the same results in simulation, while (2) is faster.

Using either method, the wavefront in our simulations is "sensed" within the image region in which we wish to create a dark hole at 5 or 6 monochromatic wavelengths sampling the bandpass. This allows a polychromatic solution to be derived, whereas sensing at just one wavelength would produce a less optimal broadband result.

The DM settings that create the dark hole are derived using the Electric Field Conjugation ${ }^{10}$ (Energy Minimization) method. This technique makes the approximation that the wavefront responds linearly to changes induced by the DMs. The effect of each DM actuator on the image plane electric field is computed at each sensing wavelength by pistoning the actuator by a small amount $(0.1-1 \mathrm{~nm})$ and propagating the effect through the model system. The electric field at the 
image plane is stored in a DM response matrix. This represents the most time-consuming portion of the simulation process as there are about 3600 active actuators total on both DMs. For some systems we can take advantage of symmetry and compute the responses for a fraction of the actuators.

The wavefronts at the sensing wavelengths and the DM response matrix form a linear system that can be solved in a least-squares sense. Because the system is not truly linear, this process must be iterated. Regularization is used to limit the actuator strokes to the linear regime, with the optimal regularization value achieved through trial-and-error.

Once a suitable dark hole has been generated, other aberrations can be introduced to determine the sensitivity of the system to the effects of pointing errors and thermally-induced wavefront changes.

\section{BANDLIMITED LYOT CORONAGRAPH}

\subsection{Modeling}

The Lyot coronagraph used in this study has a bandlimited mask that varies both the phase and amplitude in the image plane. The wavefront is propagated to the intermediate image plane and multiplied by the complex-valued occulter pattern. The beam is then propagated to a pupil image where the Lyot stop is applied. The stop is a simple aperture smaller than the pupil diameter and matched to the modified pupil pattern created by the occulter. The simplicity of the Lyot coronagraph allows it to be easily and accurately modeled using common propagation algorithms.

\subsection{Caveats}

Propagation through the Lyot coronagraph utilizes standard techniques like those used in PROPER. Models of bandlimited Lyot coronagraphs have been compared to results from HCIT at contrast levels equal to those required for exo-Jupiter imaging $\left(10^{-9}\right.$ contrast). The simulated and observed results match very well, and the models have been used to guide experiments. The major expected source of inaccuracies in the modeling results is that the occulter phase and amplitude transmissions in the models are wavelength independent (e.g., the same phase shift in radians is applied at each wavelength). A real occulter would have some wavelength-dependent dispersion that would reduce the performance over a broad bandpass. This can be minimized with a judicious choice of dielectric coatings. Predicted dispersions based on multilayer coating calculations will be applied in future modeling efforts.
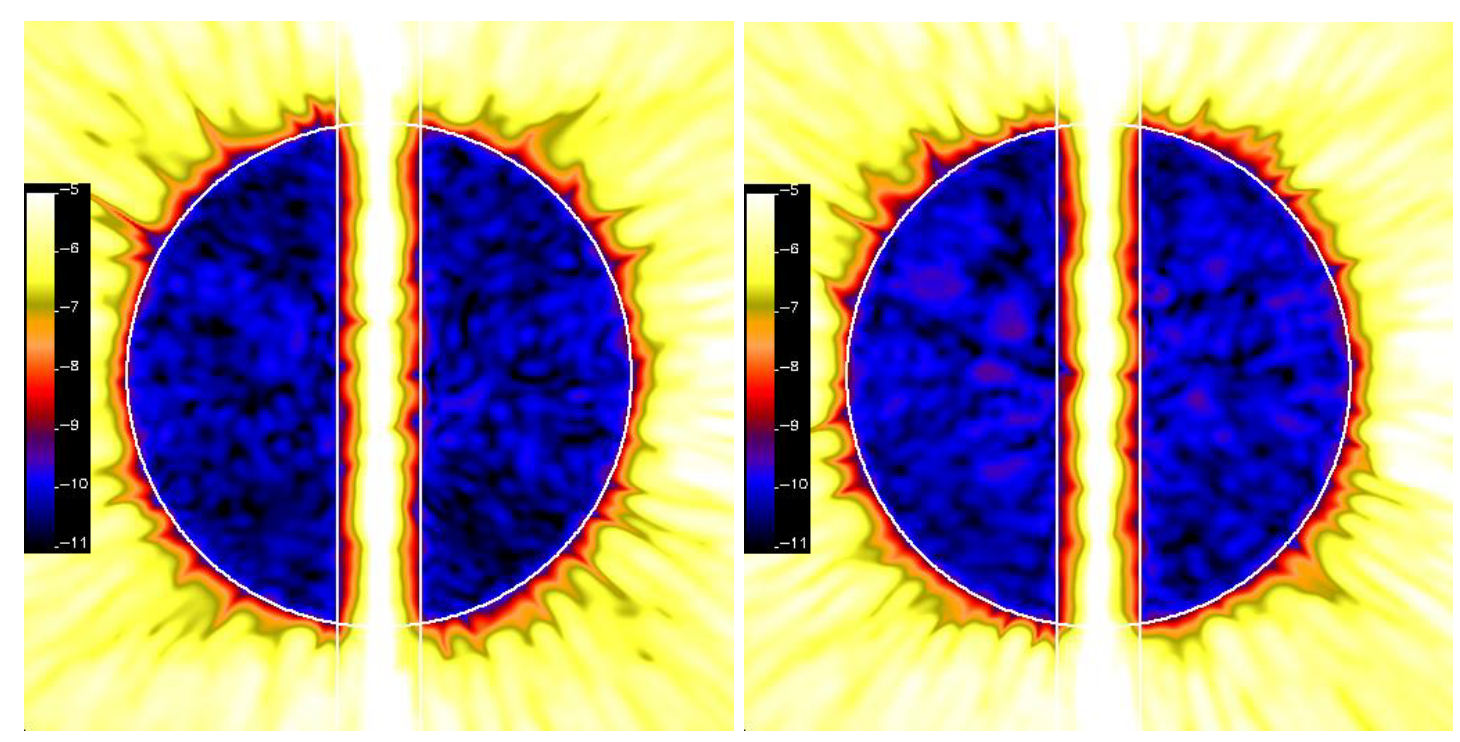

Fig. 4. Contrast maps for a hybrid occulter Lyot coronagraph over $\lambda=500-600 \mathrm{~nm}$ after wavefront correction. A linear occulter was used. The color scale indicates $\log _{10}$ (contrast). The outer radius of $20 \lambda / \mathrm{D} @ \lambda=500 \mathrm{~nm}$ and inner radius of $3 \lambda / \mathrm{D} @ \lambda=550 \mathrm{~nm}$ are outlined. The left map shows the results for a system with $0.25 \mathrm{~nm}$ RMS surface error nonprimary optics, and the right shows the contrast with $1.25 \mathrm{~nm}$ RMS non-primary optics. The primary has $8 \mathrm{~nm}$ RMS surface errors. 


\subsection{Results}

The hybrid bandlimited occulter Lyot coronagraph, as modeled, easily meets the contrast requirement for a system having non-primary-mirror optical surface errors of $<1.25 \mathrm{~nm}$ RMS (Figure 4). It appears to allow for the correction larger wavefront errors over a broad bandpass than is possible with PIAA. This is may be due to the particular structure of the actuator response function as seen in the final image plane along with the ability to use actuators located behind the Lyot stop.

\subsection{Future modeling studies}

The wavelength-dependent amplitude and phase terms of the occulter will need to be included in future models. These must be computed from the known properties of the metal and dielectric coatings used in the occulters. These deviations from the ideal wavelength-independent model used in this study may result in lower contrast dark holes or, in order to maintain the current contrast levels, reduced bandwidth. Manufacturing errors (e.g., deviations from the desired amplitude profile) should also be included (these have in previous studies for HCIT experiments).

\section{OPTICAL VORTEX CORONAGRAPH}

\subsection{Modeling}

The OVVC effectively produces a phase ramp through fractional manipulation of the polarization of the incoming wavefront. Rather than duplicating the physics involved in this process, it is easier to simulate its result, the phase screw, when modeling wavefront propagation through the system. Even so, the fact that there is a numerical singularity at the center of the screw causing rapid phase changes that cannot be exactly represented on the computational grid means that additional steps must be taken to produce a sufficiently accurate simulation.

The image of the PSF in the plane of the vortex mask must be highly oversampled to match the fine sampling required to adequately represent the phase screw. This is accomplished by using a small pupil diameter relative to the size of the computational wavefront array. In the OVVC simulations, the pupil diameter is 204.8 pixels while the grid diameter is 2048 pixels, producing a PSF that is sampled by $0.1 \lambda / D$ (5 times better than the Nyquist criterion).

In the representation of the phase screw, the central 127 by 127 pixel region, including the occulting spot, is generated on a grid subsampled by a factor of 51 in each direction (6477 pixels on a side) and then rebinned to the wavefront sampling. The outer regions of the mask are directly computed. At the subsequent Lyot plane, the reimaged pupil is masked by a circular stop with a $90 \%$ clear diameter.

Both the OVVC and Lyot coronagraphs can be modeled using PROPER and its default propagation algorithms. Both can utilize the same optical prescription and surfaces.

As discussed in the OVVC description earlier in this document, the design results in a "leakage" of unsuppressed PSF light that is incoherent with the residual field. To approximate this term, a PSF is generated by propagation through the system without the image plane mask. This PSF is then normalized by a fractional leakage factor and added in intensity to the coronagraphic field.

\subsection{Caveats}

The OVVC is a relatively new concept and to date only one such mask has been tested in the HCIT. Thus, only a limited amount of model verification has been done, none at the contrast levels required for exoplanet imaging. The models agree with analytical calculations, and expectations are that the models produced using the phase screw described above are accurate to better than $10^{-9}$ contrast.

\subsection{Results}

The OVVC was simulated using PROPER for both 10\% (525-575 $\mathrm{nm})$ and 20\% (500-600 nm) bandpasses (Figure 5). The inner working angle was $2 \lambda / D$ at $\lambda=550 \mathrm{~nm}$. The occulting spot was $0.25 \lambda / D$ in radius. Using EFC, dark hole fields were generated for each case. The $20 \%$ bandpass simulation has a mean contrast value of $1.9 \times 10^{-10}$ over the entire hole and $2.9 \times 10^{-9}$ at $r=2-3 \lambda / D$. With a $10 \%$ bandpass, the mean contrast is $5.0 \times 10^{-11}$ and $1.3 \times 10^{-9}$ at $2-3 \lambda / D$. In both cases the results represent fields observed at one polarization. The models show that the OVVC, as simulated, can 
achieve the required dark field over a $10 \%$ bandpass and also at $20 \%$, though the region near the IWA is slightly higher than desired.

\subsection{Future modeling studies}

More accurate representation of the wavelength-dependent terms is needed to provide a better estimate of the broadband performance of the OVVC. Polarization effects also need to be examined in greater detail. Specifically, simulations are needed to determine if it is possible to create a sufficiently deep dark hole in both polarizations using one pair of DMs (rather than having two separate polarization channels, each with its own DM pair, which would increase cost and complexity).

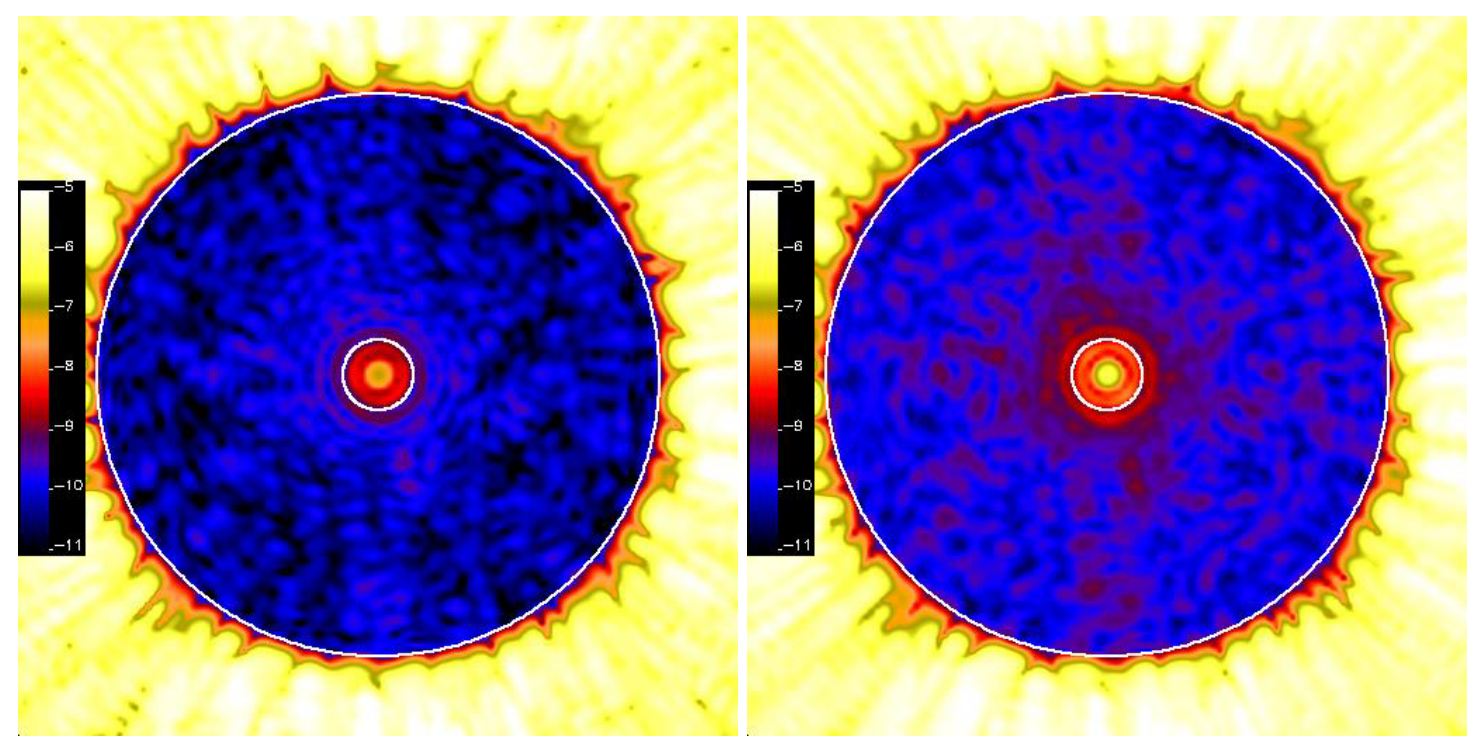

Fig. 5. Contrast maps for OVVC after wavefront correction. The color scale indicates $\log _{10}$ (contrast). The outer radius of 20 $\lambda / \mathrm{D} @ \lambda=500 \mathrm{~nm}$ and inner radius of $2 \lambda / \mathrm{D} @ \lambda=550 \mathrm{~nm}$ are outlined. The left map shows the results for a system operating at $\lambda=525-575 \mathrm{~nm}$ (10\% bandpass) and on the right is $\lambda=500-600 \mathrm{~nm}(20 \%$ bandpass $)$.

\section{PHASE INDUCED AMPLITUDE APODIZATION (PIAA)}

\subsection{Modeling}

Simulating propagation between the two PIAA mirrors presents a considerable challenge. To produce strong beam apodization, the surface curvature rapidly changes near the outer edge of the first PIAA mirror, introducing a large wavefront variation that cannot be adequately sampled using conventional Fourier-based algorithms without impractically large arrays. Three alternative algorithms have been devised:

S-Huygens: Vanderbei ${ }^{11}$ derived a method to accurately compute propagation within the PIAA system called SHuygens. It is the only method presented here that includes the effects of diffraction. Belikov et al. ${ }^{12}$ described the application of S-Huygens for propagating wavefronts that can be decomposed into radially-weighted azimuthal harmonics. We have developed code that can propagate an arbitrary wavefront through PIAA with this method. However, the number of harmonics needed to sufficiently describe the wavefront for this study is about 450 (to adequately sample a feature at the edge of the pupil that has a diameter $\sim 1 / 50^{\text {th }}$ of the pupil). A multithreaded version of the code running on an 8 processor system takes one hour to propagate a monochromatic wavefront between the optics in both the forward and reverse PIAA systems (propagation from/to the PIAA systems is done using conventional propagation algorithms). This makes S-Huygens impractical for routine computations, as thousands of separate wavefront propagations are needed to compute the DM response matrix. 
Geometric remapping with propagation of harmonics: This method approximates the propagation of a harmonic wavefront error (i.e., a ripple in amplitude or phase) between the two PIAA mirrors. By separately propagating different harmonics and then adding the results, the propagation of a more complex wavefront aberration can be simulated with Talbot effects included. This technique, however, completely ignores diffraction by assuming that its effects are sufficiently suppressed using a post-PIAA apodizer. Propagation of an arbitrary wavefront is a three-stage process. First, the wavefront is decomposed into its component harmonics by computing its Fourier transform. Second, the complexvalued, two-dimensional representation of the wavefront error described by that particular harmonic is then geometrically distorted to represent remapping by the PIAA optics and an apodization function applied to represent the combined PIAA and post-apodizer system. Finally, the distorted wavefront is multiplied by two phase terms, one that propagates the wavefront over some distance while the other, which is dependent on the spatial frequency of the aberration, replicates the Talbot effect. This method is somewhat faster than S-Huygens. The greatest drawback is that it propagates only pure harmonic signals that are assumed to have infinite extent. A decomposition of a pupil-bounded wavefront into harmonics will result in "impure," finite-extent harmonics that contaminate the propagator and create artifacts. Unless this problem is solved, perhaps by deconvolution of the aberration frequency spectrum, we cannot use this method except when manually specifying individual spatial frequency aberrations. This method is also too slow to be practical for this study.

Geometric remapping only: This is the simplest method. The wavefront is distorted (Figure 6) and then multiplied by the PIAA and post-apodizer apodization functions. This completely ignores both diffraction and Talbot effects. It is very quick, however, and captures some of the important effects caused by remapping.

Due to time constraints the third method is used for propagation between the two PIAA mirrors in both the forward and reverse PIAAs. The wavefront up to the first PIAA mirror (PIAA M1) is computed using the default PROPER routines. The measured surface error map of the Tinsley M1 optic is applied. The wavefront is then remapped via cubic convolution interpolation. Care must be taken to ensure that pixels at the edge of the pupil-bounded wavefront are interpolated accurately. The error map for the M2 Tinsley mirror is then applied. The wavefront is then multiplied by a function representing the apodization provided by the first PIAA optic as seen at the second optic. The wavefront is then propagated to a mirror (apodizer OAP) that then creates an image of the $2^{\text {nd }}$ PIAA mirror on the post-apodizer, where another apodization function is applied. There is then a propagation to another mirror (occulter OAP) that focuses the beam onto the occulter, which is represented by a solid disk. After that the beam is propagated to a mirror that then feeds the reverse PIAA system. In the reverse system the wavefront is again interpolated to undistort the wavefront and the apodization provided by the forward PIAA optics (but not the post-apodizer) is divided out. From there on the beam is propagated through other optics to the final image plane.

Aberration before PIAA

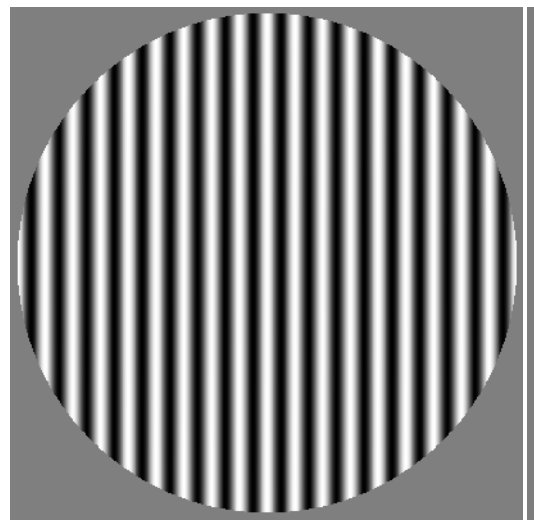

Aberration after PIAA

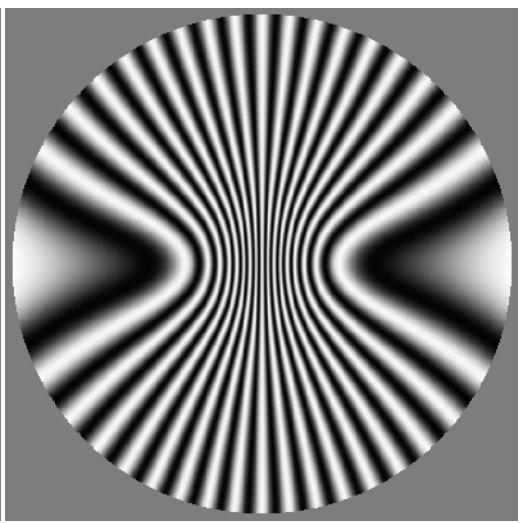

Fig. 6. Demonstration of the wavefront remapping performed by the forward PIAA optics using phase ripple aberration. This distortion is corrected by the reverse PIAA optics

\subsection{Caveats}

A number of approximations must be made when modeling PIAA with an arbitrary input wavefront, especially with time constraints of this study. With the geometric remapping method, diffraction at the edge of the $1^{\text {st }}$ PIAA optic is not 
accounted for. This likely results in an underestimation of the amount of unsuppressed PSF signal that leaks into the dark hole, a problem that is probably highly chromatic and difficult to remove using the DMs. In the models surface errors on the PIAA optic do not alter the apodization function, as they would in reality (including creating chromatic amplitude errors). The conversion of phase-to-amplitude errors and vice-versa by the Talbot effect are not handled. All of these will limit the accuracy of the broadband response of the system. Comparisons with more sophisticated methods (namely S-Huygens) have not yet been made for complex wavefronts.

None of the propagation methods have been verified against results obtained with actual PIAA optics at contrast levels approaching the planet imaging requirements.
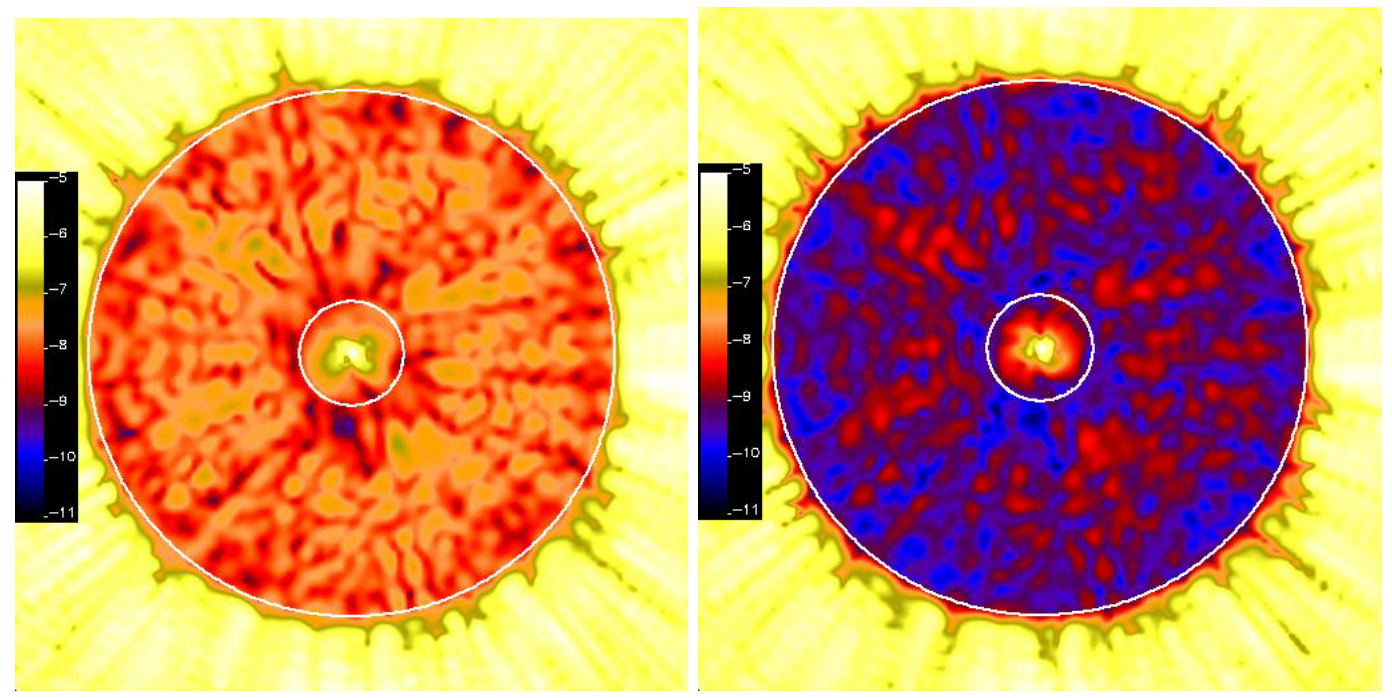

Fig. 7. Contrast maps for a simulated PIAA system over $\lambda=500-600 \mathrm{~nm}$ after wavefront correction. The color scale is given in $\log _{10}$ (contrast). The outer radius of $20 \lambda / \mathrm{D} @ \lambda=500 \mathrm{~nm}$ and inner radius of $3 \lambda / \mathrm{D} @ \lambda=550 \mathrm{~nm}$ are outlined. In both cases the primary mirror had $8 \mathrm{~nm}$ RMS surface error. The left map shows the contrast limit when all of the nonprimary optics have $1.25 \mathrm{~nm}$ RMS surface errors (mean contrast $=1.8 \times 10^{-8}$ ), and on the right when the surface errors on just the post-PIAA OAPs and near-focus flats are reduced to $0.25 \mathrm{~nm}$ RMS (mean contrast $=8.3 \times$ $\left.10^{-10}\right)$.

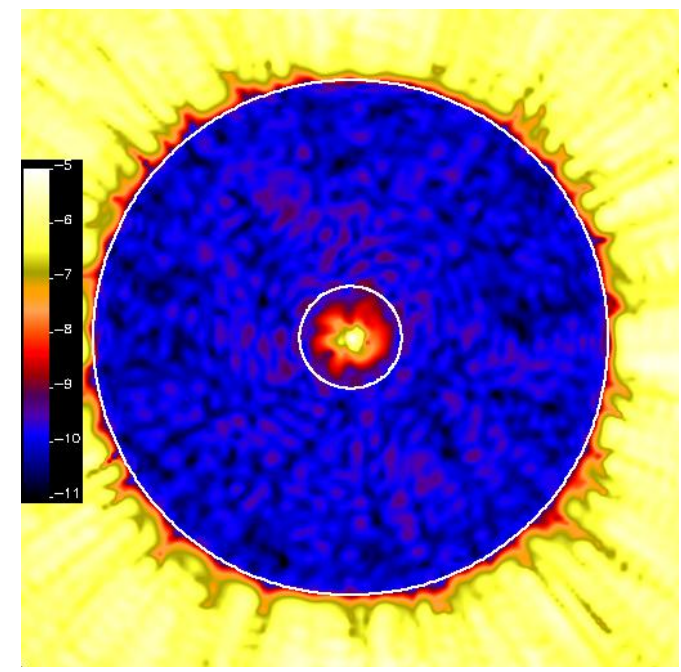

Fig. 8. Contrast map with same parameters as those in Figure 7 but with perfect post-PIAA OAPs and near-focus optics (all other non-primary optics are $1.25 \mathrm{~nm}$ RMS surface). 


\subsection{Results}

To the level of accuracy provided by the geometric remapping method, the PIAA system as implemented in this study can achieve a mean dark hole contrast of $8.3 \times 10^{-10}$ in a $20 \%$ bandpass, though some speckles as bright as $5 \times 10^{-9}$ in contrast occur (Figure 7). This level can only be achieved with certain critical optics having extremely low surface errors $(<0.25 \mathrm{~nm}$ RMS $)$. Specifically, these are the two fold mirrors prior to the occulter that are located near a focus and the two OAPs between the forward PIAA optics and occulter. The contrast requirement is not reached if any of these four optics has surface errors of $>0.5 \mathrm{~nm}$ RMS, while the other non-primary-mirror optics in the system can have $1.25 \mathrm{~nm}$ RMS surface errors (Figure 8).

The residual high level speckles are highly chromatic and are brightest at the shortest wavelength. It has been demonstrated that optics near focus must be very smooth or else the phase errors they introduce will turn into chromatic amplitude errors that cannot be fully corrected ${ }^{8}$. The aberrations on the post-PIAA OAPs appear to the DMs and the detector (both located outside of the distorted PIAA image space) as highly magnified towards the center of the pupil and compressed near the edge. High spatial frequency aberrations on the OAPs appear as a combination of much lower frequency ones that the DMs can correct and higher frequency errors that it cannot ${ }^{13}$. These residual aberrations, through frequency folding, may create highly chromatic scattered light inside the dark hole that cannot be corrected by the DMs. The effects of such errors will require further study.

It appears that it is more difficult to achieve a broadband dark hole with a PIAA system than with a bandlimited Lyot coronagraph given the same optical surface error levels. PIAA does not appear to have the same leverage to correct chromatic aberrations as the Lyot coronagraph has with its particular actuator response functions and actuators masked by the Lyot stop.

\subsection{Future modeling studies}

PIAA requires a significant advancement in modeling techniques in order to reach the same levels of simulation accuracies as are possible for the other coronagraphs. While S-Huygen can provide the accuracy, it is too slow to be useful for end-to-end modeling (it is useful for limited comparisons with other techniques, though). Comparisons of the other methods (remapping, remapping with harmonics) to S-Huygens and to testbed results are needed. Hopefully, some new method will be devised that provides the accuracy of S-Huygens but with considerably improved speed.

The apparent difficulty that PIAA has dealing with chromatic errors introduced by near-focus or post-PIAA optics needs further analysis. The surface requirements suggested by this study are at the extreme limits of current technology, and attempts to get contrast down to the $10^{-10}$ level required for an exo-Earth mission (rather than an exo-Jupiter mission) appear unlikely. This sensitivity of PIAA in the models needs to be verified against testbed results with a similar configuration (DMs prior to the PIAA optics, inverse PIAA optics included).

\section{ACKNOWLEDGEMENTS}

The research described in this publication was carried out at the Jet Propulsion Laboratory, California Institute of Technology, under a contract with the National Aeronautics and Space Administration. Work was performed as part of the ACCESS mission concept study and the Exoplanet Exploration Program at JPL.

\section{REFERENCES}

[1] Kuchner, M. J., Traub, W. A., "A coronagraph with a band-limited mask for finding terrestrial planets," Astrophysical Journal, 570, 900 (2002).

[2] Moody, D. C., Trauger, J. T., "Hybrid Lyot coronagraph masks and wavefront control for improved spectral bandwidth and performance," Proc. SPIE, 6693, 66931I (2007).

[3] Palacios, D. M., “An optical vortex coronagraph,” Proc. SPIE, 5905, 196 (2005).

[4] Mawet, D., Serabyn, E., Liewer, K., Hanot, C., McEldowney, S., Shemo, D., O’Brien, N., "Optical vectorial vortex coronagraphs using liquid crystal polymers: theory, manufacturing, and laboratory demonstration," Optics Express, 17, 1902 (2009). 
[5] Lee, J. H., Foo, G., Johnson, E. G., Swartzlander Jr., G. A., "Experimental verification of an optical vortex coronagraph," Physical Review Letters, 97, 53901 (2006).

[6] Guyon, O., Pluzhnik, E. A., Galicher, R., Martinache, F., Ridgway, S. T., Woodruff, R. A., "Exoplanet imaging with a phase-induced amplitude apodization coronagraph: I. Principle," Astrophysical Journal, 622, 744 (2005).

[7] Goodman, J., Introduction to Fourier Optics (2 ${ }^{\text {nd }}$ Edition), McGraw-Hill Companies, Inc. (1996).

[8] Shaklan, S. B., Green, J. J., "Reflectivity and optical surface height requirements in a broadband coronagraph," Applied Optics, 45, 5143 (2006).

[9] Krist, J. E., "PROPER: an optical propagation library for IDL," Proc. SPIE, 6675, 66750P (2007).

[10] Give'on, A., Kern, G., Shaklan, S., Moody, D., Pueyo, L., "Broadband wavefront correction algorithm for highcontrast imaging systems," Proc. SPIE, 6691, 66910A (2007).

[11] Vanderbei, R. J., "Diffraction analysis of two-dimensional pupil mapping for high-contrast imaging," Astrophysical Journal, 636, 528 (2006).

[12] Belikov, R., Kasdin, N., Vanderbei, R. J., "Diffraction-based sensitivity analysis of apodized pupil-mapping systems," Astrophysical Journal, 652, 833 (2006).

[13] Give'on, A., Kasdin, N. J., Vanderbei, R. J., Avitzour, K., "On representing and correcting wavefront errors in highcontrast imaging systems," J. Opt. Soc. Am. A., 23, 1063 (2006). 